\title{
Sub-Picosecond Tunable Hard X-Ray Undulator Source for Laser/X-Ray Pump-Probe Experiments
}

\author{
G. Ingold, P. Beaud, S. Johnson, A. Streun, T. Schmidt, R. Abela, A. Al-Adwan, D. \\ Abramsohn, M. Böge, D. Grolimund, A. Keller, F. Krasniqi, L. Rivkin, M. Rohrer, \\ T. Schilcher, T. Schmidt, V. Schlott, L. Schulz, F. van der Veen, D. Zimoch \\ Swiss Light Source, Paul Scherrer Institut, CH-5232 Villigen, Switzerland
}

\begin{abstract}
The FEMTO source under construction at the $\mu$ XAS beamline is designed to enable tunable time-resolved laser/xray absorption and diffraction experiments in photochemistry and condensed matter with ps- and sub-ps resolution. The design takes advantage of (1) the highly stable operation of the SLS storage ring, (2) the reliable high harmonic operation of small gap, short period undulators to generate hard x-rays with energy 3-18 keV at $2.4 \mathrm{GeV}$ beam energy, and (3) the progress in high power, high repetition rate fs solid-state laser technology to employ laser/e-beam 'slicing' to reach a time resolution of ultimately $100 \mathrm{fs}$. The source will profit from the inherently synchronized pump (laser I: $100 \mathrm{fs}, 2 \mathrm{~mJ}, 1 \mathrm{kHz}$ ) and probe (sliced X-rays, laser II: $50 \mathrm{fs}, 5 \mathrm{~mJ}, 1 \mathrm{kHz}$ ) pulses, and from the excellent stability of the SLS storage ring which is operated in top-up mode and controlled by a fast orbit feedback (FOFB). Coherent radiation emitted at THz frequencies by the sliced $100 \mathrm{fs}$ electron bunches will be monitored as on-line cross-correlation signal to keep the laser-electron beam interaction at optimum. The source is designed to provide at $8 \mathrm{keV}(100 \mathrm{fs})$ a monochromized flux of $10^{4} \mathrm{ph} / \mathrm{s} / 0.01 \%$ bw (Si crystal monochromator) and $10^{6} \mathrm{ph} / \mathrm{s} / 1.5 \% \mathrm{bw}$ (multilayer monochromator) at the sample. It is operated in parasitic mode using a hybrid bunch filling pattern. Because of the low intensity measurements are carried out repetitively over many shots using refreshing samples and gated detectors. 'Diffraction gating' experiments will be used to characterize the sub-ps X-ray pulses.
\end{abstract}

Keywords: sub-ps X-rays, time-resolved, undulator radiation, fs laser PACS: $41.75 . \mathrm{Ht}, 41.60 . \mathrm{Ap}, 42.65 . \mathrm{Re}$

\section{INTRODUCTION}

In the presence of a static magnetic field, an electron can interact with light to either emit or absorb energy. Although the magnetic field of a tightly focused laser beam is much higher than the static one, its coupling to the electron beam is neglegible if the electron has relativistic energy $(\beta \simeq 1)$ and co-propagates with the laser beam. If the planar magnetic field of a static periodic wiggler is tuned to the wavelength of a horizontally linear polarized laser, the electron will resonantly interact with the optical electric field and - depending on relative phase - will gain or loose energy. The process is described by the theory of a small signal gain FEL amplifier [1]. More recently [2] it has been realized that the same process can be used in a storage ring to impose an energy modulation on the electron beam of several sigma beyond the natural energy spread. By using a fs laser, a sub-ps slice of an electron bunch can be generated after passing a dispersive section in the ring. To achieve sufficient energy modulation to separate the 'sliced' electrons from the core beam, the laser pulse energy should be rather high $(3-5 \mathrm{~mJ}$ at $2.4 \mathrm{GeV})$ and the number of wiggler periods matched to the number of optical laser cycles.

The 'slicing' technique first demonstrated at the ALS [3] has recently been implemented at BESSY [4] to generate sub-ps soft x-rays (1-2 keV) with variable polarization. The SLS FEMTO slicing source [5] currently under commissioning at the $\mu \mathrm{XAS}$ beamline of the SLS storage ring $(2.4 \mathrm{GeV})$ is designed to generate $\mathrm{x}$-rays in the range $3-18 \mathrm{keV}$ based on the high harmonic (11./13.) operation of a short period in-vacuum undulator [6].

The FEMTO slicing facility consists of two main parts, the FEMTO laser system and the FEMTO insertion ('slicing spectrometer'). The construction and operation of the facility take advantage of its modular design to allow laser/xray pump/probe experiments with time resolution of either $100 \mathrm{ps}$ or $0.1 \mathrm{ps}$ (slicing operation). Hybrid bunch filling ('camshaft' single pulse current: 3-8 mA) requires gated detectors (APDs, Si- $\mu$ strip- and pixel- detectors).

So far laser(100 fs)/x-ray(100 ps) pump/probe time-resolved experiments have been performed in absorption and diffraction using both solid and liquid samples. As an example, we present briefly the ps characterization of strain wave dynamics in near-surface heated crystalline InSb. 

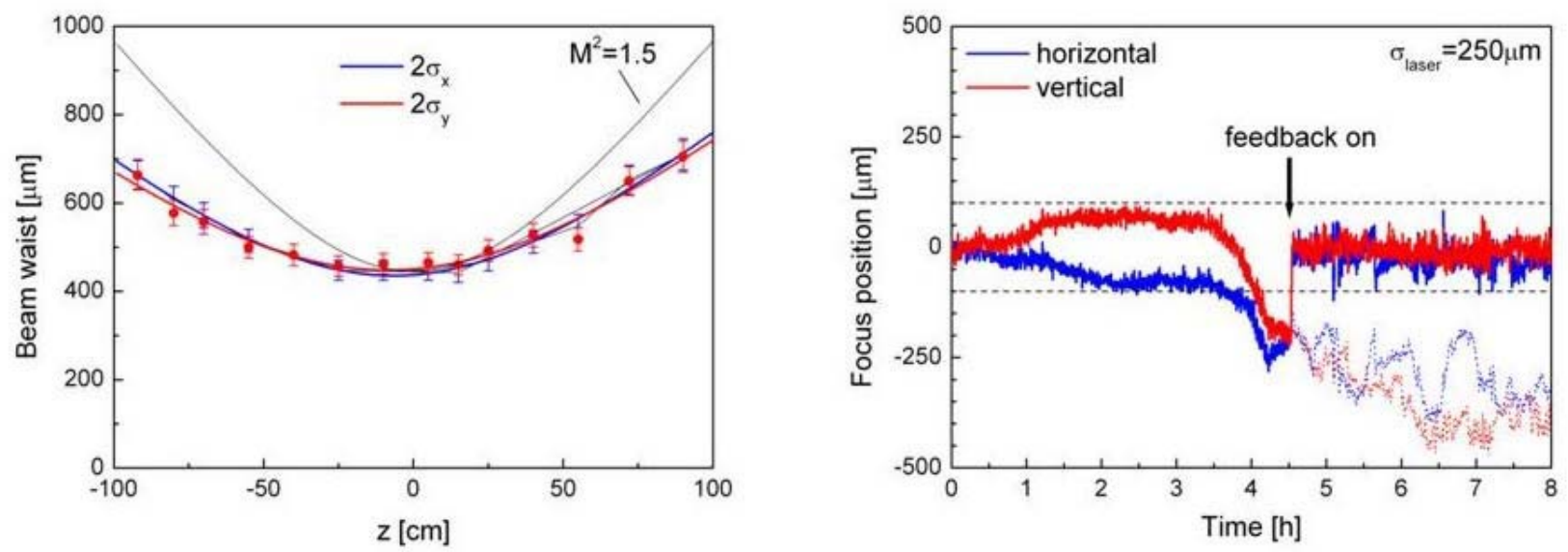

FIGURE 1. Laser amplifier II: Essential for efficient slicing is a near diffraction limited spatial profile of the laser beam and a high pointing stability at the interaction point inside the modulator.

\section{LASER SYSTEM}

A high average power femtosecond laser system has been installed near the experimental hutch of the $\mu$ XAS beamline. The laser system consists of three parts: (1) a fs oscillator ( $30 \mathrm{fs}, 100 \mathrm{MHz}, 500 \mathrm{~mW}, 795 \mathrm{~nm}$ ) that is synchronized to the rf-master oscillator $(500 \mathrm{MHz})$ with a jitter estimated to be $<1 \mathrm{ps},(2)$ a 'phase I' regenerative amplifier (115 fs, $1 \mathrm{kHz}, 2.1 \mathrm{~mJ}, 800 \mathrm{~nm}$ ) for pumping experimental samples, and (3) a 'phase II' amplifier system (consisting of a regenerative amplifier and a symetrically pumped 2-pass amplifier: $50 \mathrm{fs}, 1 \mathrm{kHz}, 5.2 \mathrm{~mJ}, 805 \mathrm{nmm}$ ) that is used to produce sub-ps x-rays within the storage ring. The Ti:sapphire laser crystal of the amplifier is cryogenically cooled to $45 \mathrm{~K}$ to ensure a good mode quality $\left(\mathrm{M}^{2} \leq 1.1\right)$ of the outgoing laser beam.

A 5 "-aperture magnifying telescope has been set up in the laser hutch to focus the laser in vacuum over an optical distance of $\sim 45 \mathrm{~m}$ into the modulator where the laser-electron interaction (slicing) takes place. This configuration was chosen to maintain the good spatial and temporal quality of the laser beam by minimizing phase distortions of the intense fs laser pulses, which interact nonlinearly with optical media such as windows, lenses and air.

Special attention was given to the rigidity and accuracy of the mirrors that are placed inside the tunnel to direct the laser into the modulator. These mirrors are placed inside the UHV of the storage ring and are remote controlled. Cameras are used for optical alignment, diagnostics and active beam stabilization (see Figure 1).

\section{SLICING SPECTROMETER}

To generate sub-ps undulator radiation above $3 \mathrm{keV}$ at $2.4 \mathrm{GeV}$, small gap operation of both the modulator and the radiator is needed. To minimize bunch lengthening effects, the FEMTO insertion has been installed in the long straight section 5L of the SLS storage ring. The sequence of magnets act as a 2-stage spectrometer: the energy-modulated satellite electrons ('secondary beam') generated in the modulator — due to the dispersion provided by a chicane - are separated from the core beam inside the radiator, where the $\mathrm{x}$-rays are generated.

A new (FEMTO) quadrupole triplet has been installed to match the beta- functions for the modulator and the radiator (beam stay clear aperture of $8 \mathrm{~mm}$ and $5 \mathrm{~mm}$, respectively) installed at the entrance and exit of straight $5 \mathrm{~L}$. This break of the 3-fold ring symmetry does not affect the dynamic acceptance of the SLS storage ring.

For sub-ps operation the modulator (hybrid wiggler W138: $\mathrm{B}_{\text {eff }}=1.98 \mathrm{~T}, \mathrm{~g}=11 \mathrm{~mm}, \lambda_{w}=138 \mathrm{~mm}, 16$ periods) is operated at resonance, close to the amplifier II laser wavelength of $805 \mathrm{~nm}$. A 3-dipole chicane provides horizontal spatial and angular dispersion of $0.44 \mathrm{~m}$ and $0.1 \mathrm{rad}$, respectively, to separate the satellite and core electron beams at the center of the radiator. The radiator is a small gap, short period hybrid in-vacuum undulator operated at high harmonics ( $\mathrm{B}_{e f f}=0.94 \mathrm{~T}, \mathrm{~g}=5 \mathrm{~mm}, \lambda_{u}=19 \mathrm{~mm}, 96$ periods). For $400 \mathrm{~mA}$ (390 bunches) the measured monochromatic flux at $8 \mathrm{keV}$ at the sample is $8 \cdot 10^{12} \mathrm{ph} / \mathrm{s} / 0.01 \%$ bw (aperture $240 \times 54 \mu \mathrm{rad}^{2}$ ). Using a $5 \mathrm{~mA}$ 'camshaft pulse' of 50 ps (FWHM) (using factor 2 bunch shortening by detuning the SC 3rd harmonic cavity), the expected sliced flux at 8 


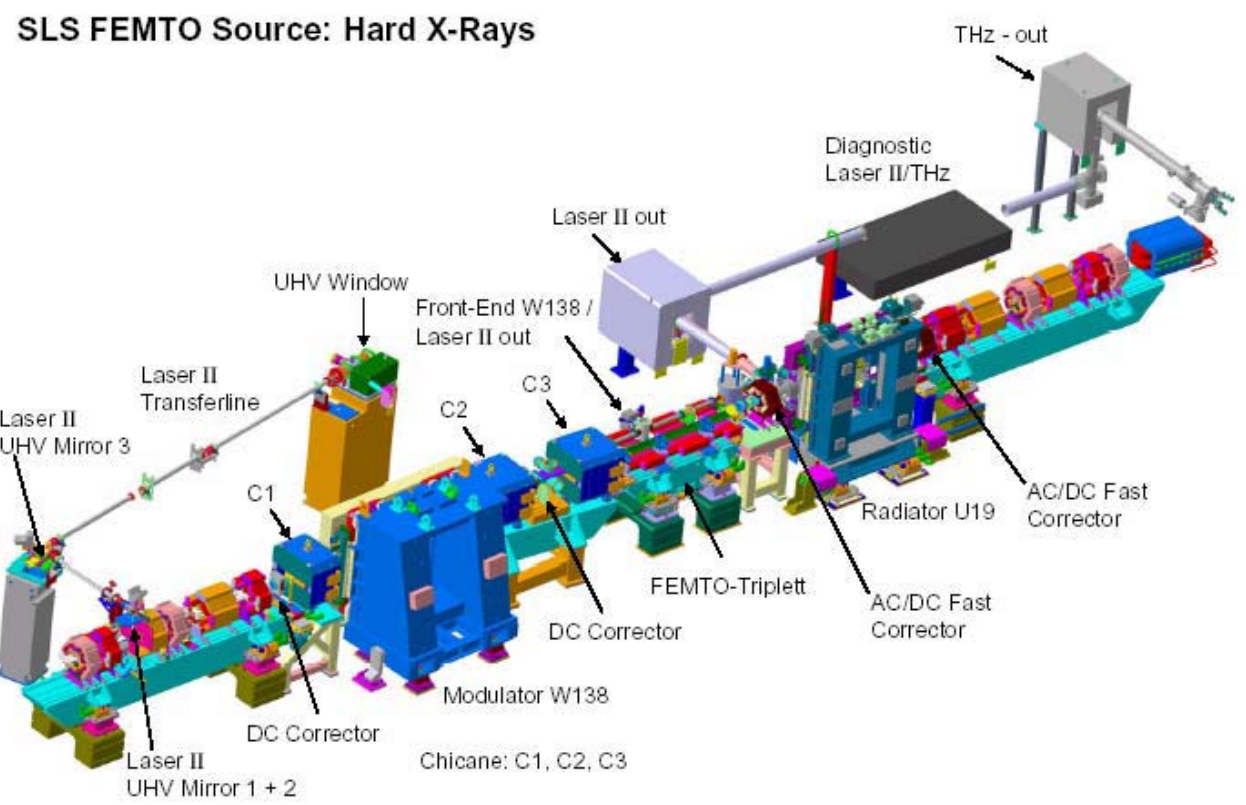

FIGURE 2. Engineering layout of the FEMTO source: Laser II transport line, slicing spectrometer installed in a long (11 $\mathrm{m}$ ) straight section, and ports for laser and THz diagnostic.
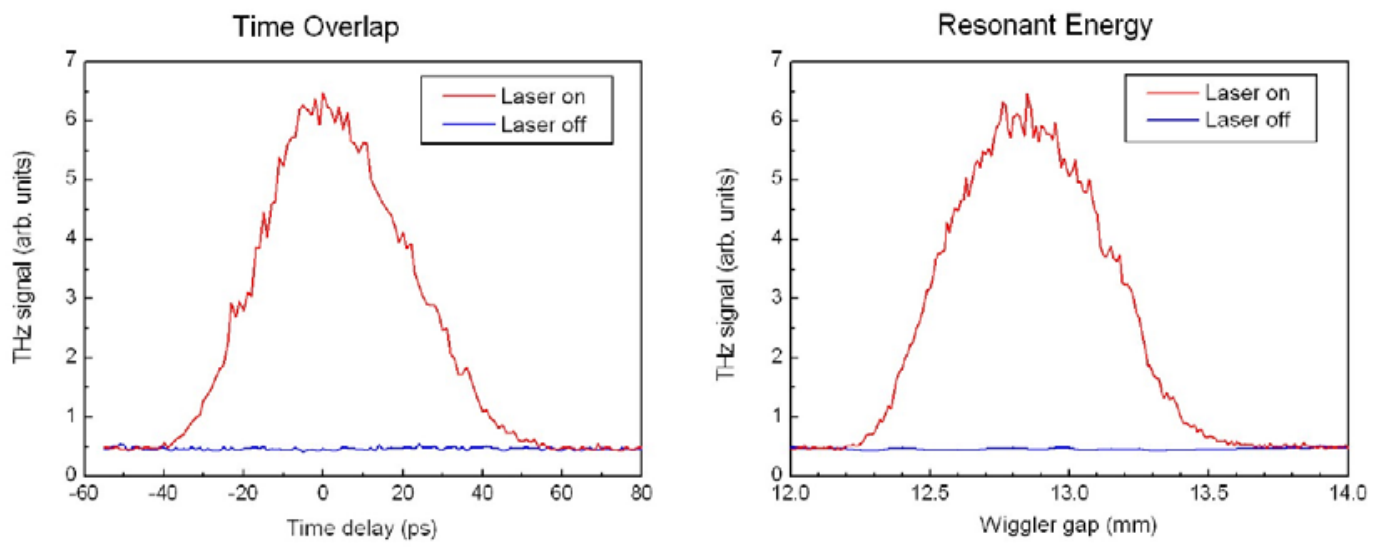

FIGURE 3. Measured coherent THz-radiation emitted by fs laser sliced electrons.

$\mathrm{keV}$ at the sample is $10^{4} \mathrm{ph} / \mathrm{s} / 0.01 \% \mathrm{bw}$ (Si crystal monochromator) and $10^{6} \mathrm{ph} / \mathrm{s} / 1 / 5 \%$ (multilayer monochromator). Corrector magnets and beam position monitors both for the electron and x-ray beam have been installed to control residual orbit distortions in position and angle to ultimately $1 \mu \mathrm{m}$ and $1 \mu \mathrm{rad}$, respectively, when feedforward (FF) and fast orbit feedback (FOFB) corrections are applied.

To suppress background radiation, the modulator is tilted by $2.4^{\circ}$ with respect to the radiator center. Spectrum calculations including all possible background contributions (namely $\mathrm{x}$-rays from the up- and downstream bending magnets, from the chicane dipole magnets $\mathrm{C} 1-\mathrm{C} 3$ and from the modulator) show that $\mathrm{x}$-rays emitted from sliced electrons with energy modulation $\Delta \mathrm{E} / \mathrm{E} \geq 0.5 \%$ can be separated using a slit system installed at the $\mu \mathrm{XAS}$ beamline in $15 \mathrm{~m}$ distance from the radiator. Because the $\mathrm{x}$-rays do not pass windows or any optical element in front of the slit, the possibly harmful background is entirely due to the magnetic elements. To achieve a signal-to-background ratio of 10:1, several photon absorbers have been installed along the spectrometer. 


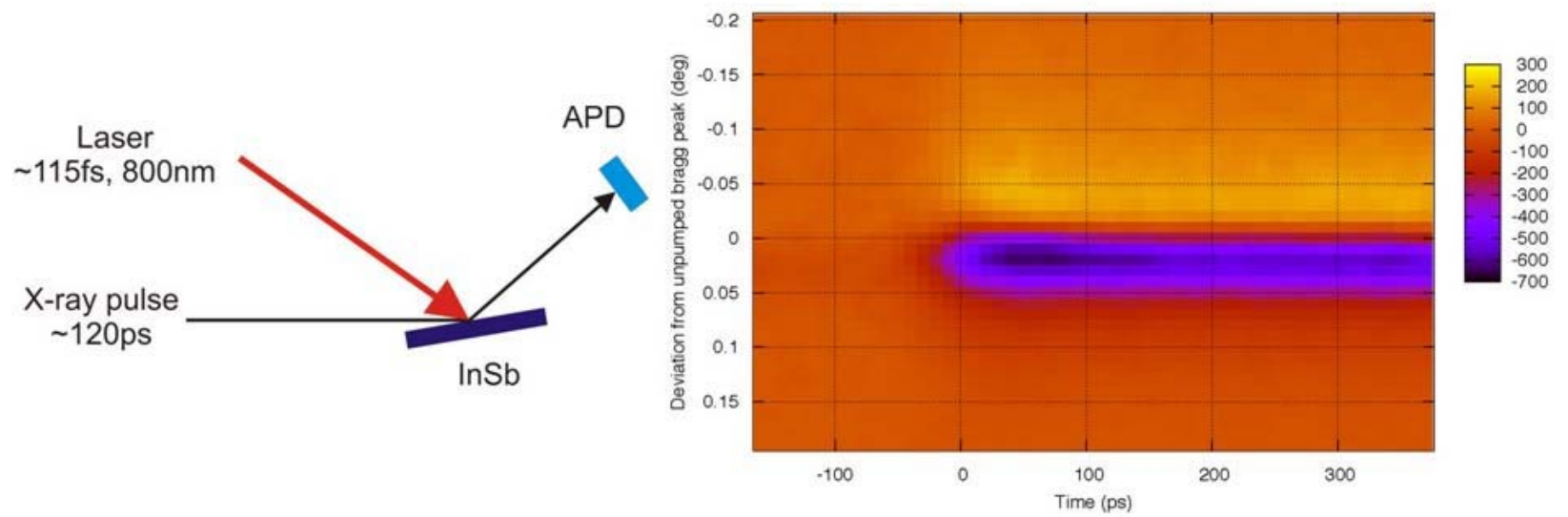

FIGURE 4. Ultrafast X-ray diffraction as a timing diagnostic: ps strain wave dynamics in a surface-heated InSb crystal. The laser strikes the surface at $0 \mathrm{ps}$, resulting in the formation of an expansion layer over the $\mathrm{x}$-ray probe depth. To resolve the dynamics within the probe depth $(<30 \mathrm{ps})$, sub-ps X-rays will be used in future experiments.

\section{SUB-PS DIAGNOSTICS}

Electrons. Coherent synchrotron radiation (CSR) in the $\mathrm{THz}$ spectral range is used as on-line diagnostic to optimize the laser- electron interaction for optimum energy modulation [7]. The CSR is due to the longitudinal charge density modulation on the $100 \mu \mathrm{m}$ length scale and is extracted at the first bending magnet downstream of the slicing spectrometer. As shown in Figure 3, the THz-intensity measured with an InSb-bolometer $\left(4.2^{\circ} \mathrm{K}\right.$, rise time $\left.0.3 \mu \mathrm{s}\right)$ is clearly correlated when scanning the relative laser-electron timing and matching the resonant energy by changing the magnetic field of the modulator via gap scan.

X-rays. There is no routine method yet to characterize the time structure of sub-ps hard X-rays. For commissioning the timing and pulse length of the slicing source, we are currently preparing an experiment to measure ultrafast diffraction from coherent phonons in InSb and Bi crystals. This experiment [8] is both adequately reversible (i.e. it can be carried out repetitively over hours) and large enough in amplitude to be observable with the sub-ps photon flux expected from this source. In a preliminary experiment we have studied the transient thermal strain in a laser-heated asymmetric cut InSb crystal with ps time resolution shown in Figure 4. "Crossed beam" experimental geometry will allow simultaneous collection of different pump-probe delay times. Gateable 1-D Si $\mu$-strip and 2-D pixel [9] detector modules under development at PSI will be used to resolve the X-ray image.

\section{REFERENCES}

1. A. Amir and Y. Greenzweig, Phys. Rev. A 34, 4809 (1986).

2. A. A. Zholents and M. S. Zoloterev, Phys. Rev. Lett. 76, 912 (1996).

3. R. W. Schoenlein et al., Science 287, 2237 (2000); R. W. Schoenlein et al., Appl. Phys. B71, 1 (2000).

4. S. Khan et al., Proceedings PAC 2005, Knoxville, 2309 (2005).

5. G. Ingold et al., Proceedings PAC 2001, Chicago, 2656 (2001).

6. G. Ingold et al., these Proceedings.

7. K. Holldack et al., Phys. Rev. Lett. 96, 054801 (2006).

8. A. M. Lindenberg et al., Phys. Rev. Lett. 84, 111 (2000); A. Rousse et al., Nature 410, 65 (2001); K. Sokolowski-Tinten et al., Nature 422, 287 (2003).

9. B. Schmitt et al., Nucl. Instr. and Meth. A 518, 436 (2004); Ch. Brönnimann et al., J. Synchrotron Rad. 120 (2006). 